\title{
Conhecimentos, práticas e percepções de profissionais de saúde sobre o tratamento de malária não complicada em municípios de alto risco da Amazônia Legal
}

\author{
Uncomplicated malaria treatment in the Brazilian Amazon: \\ knowledge, practices and perceptions of health workers \\ in high-incidence municipalities
}

\author{
Claudia Garcia Serpa O sorio-de-Castro ${ }^{1}$ \\ Elaine Silva $\mathrm{M}$ iranda $^{1}$ \\ Ângela Esher ${ }^{1}$ \\ M ônica Rodrigues deCampos² \\ Juliana de Castro Brasil ${ }^{1}$ \\ Ana Cristina Soares Ferreira ${ }^{1}$ \\ Isabel Cristina M artins Emmerick ${ }^{1}$
}

${ }^{1} \mathrm{Núcleo} \mathrm{deAssistência}$

Farmacêutica, Escola

Nacional deSaúdePública,

Fundação O swaldo Cruz.

Rua Leopoldo Bulhões 1.480/

625, M anguinhos. 21041-

210 Rio deJaneiro RJ.

claudia.osorio@ensp.fiocruz.br

${ }^{2}$ Departamento deCiências

Sociais, Escola Nacional de

SaúdePública, Fundação

Oswaldo Cruz.
Abstract Malaria control in Brazil is based on early diagnosisand adequateand timely treatment asstrategiesfor a rapid and long-lasting cure. Clinical consequences and resistance to antimalarials may arise from problems in prescribing, dispensing and in acceptance of therapeutic regimens by healthcare workers. We studied knowledge and practices, perceptionsand attitudes of health workers participating in pharmaceutical services for malaria, regarding the official protocol and the possible flaws in therapy. H ealth workers from six municipalities in the Brazilian Amazon were interviewed. Speech analysi swasemployed asa technique to determine analytical categories and to organize data. There was only 1 physician among the 63 interviewees, the others were health technicians carrying out diagnosis, therapy indication and dispensing of antimalarial treatment. Training time and period since course completion varied. Flaws in the adherence to the national protocol included therapy indication, dispensing and counseling. Health workers need knowledge to face disease and treatment specificities. H olding accountable health workers that are unprepared and unfit for the job may indicate the need for adequacy in policies regarding adequate training and hiring of human resources.

Key words Malaria, Antimalarials, Pharmaceutical services, Healthcare workers, Brazilian Amazon
Resumo 0 controle da malária no Brasil conta com diagnóstico precoce e tratamento adequado e oportuno como estratégia para cura rápida e duradoura. Consequências clínicas e resistência aos antimaláricos podem resultar defalhas na prescrição, dispensação e aceitação dos profissionais aos esquemasterapêuticos propostos. O bjetivou-seavaliar conhecimentos, práticas, percepções e atitudes de profissionais envolvidos na assistência farmacêutica à malária, frente ao protocolo oficial e a possíveis falhas na terapêutica. Entrevistaram-se profissionais em seis municípios na Amazônia Legal. U tilizou-se técnica de análise do discurso para determinação de categorias analíticas esistematização. Dos63 entrevistados, houveapenasum médico. Os demais, de nível médio, atuavam no diagnóstico, indicação e dispensação do tratamento antimalárico. 0 tempo de formação e de treinamento foi variável. H ouvefalhasna adesão ao protocolo nacional, perpassando indicação, dispensação e orientação aos pacientes. Os profissionais carecem de conhecimento para lidar com as especifi cidades da doença e do tratamento. A responsabilização de profissionais que não possuem o preparo necessário para a atenção sugere necessidade de políticas para a adequada capaci tação e incorporação de recursos humanos.

Palavras-chave Malária, Antimaláricos, Assistência farmacêutica, Profissionais de saúde, Amazônia Legal 


\section{Introdução}

Doença parasitária mais prevalente hoje, no mundo, com sérias implicações sanitárias, a malária leva a consideráveis perdas econômicas e sociais. Estão expostas à malária dois bilhões de pessoas. Houve, apenas no ano de 2002, 515 milhões de novos casos registrados ${ }^{1}$. Dados da Organização Pan-Americana da Saúde (OPAS) revelam quea maior parte da população no continente americano mora em zonas com risco de transmissão da doença².

A maláriaéenfermidadeinfecciosa febril aguda e seu agente etiológico é um protozoário do gênero Plasmodium. São mais de 125 espécies de plasmódios, das quais quatro infectam o homem: P. vivax, P. falciparum, P ovale e P. malariae. O P. falciparum éa espécie causadora da maior parte da mortalidade por malária no mundo 3 .

0 tratamento adequado e oportuno é hojeo principal alicerce para o controle da doença etem como objetivos principais assegurar uma cura clínica rápida e duradoura; evitar a progressão da doença para as formas graves, as complicações e a morte; encurtar os episódios clínicos; reduzir a ocorrência de anemia associada à malária, as consequências da infecção palúdica placentária e a anemia materna e impedir o desenvolvimento da resistência aos medicamentos ${ }^{4}$.

Como os plasmódios têm um complexo ciclo biológico, é consenso que a malária deva ser tratada com associações de medicamentos ${ }^{5}$. 0 esquema terapêutico deve atuar em todas as fases desse ciclo. É preciso usar critérios clínicos importantes, tais como o tipo de plasmódio, a classificação clínica do paciente (se portador de malária não complicada ou malária grave) e 0 grupo ao qual o paciente pertence - criança, idoso ou grávida - que indicará a necessidade de um tratamento diferenciado.

O perfil de distribuição geográfica da doença é heterogêneo, assim como as cepas de plasmódio e os vetores. Os profissionais que atuam na assistência à malária, portanto, precisam ter conhecimento da epidemiologia local, dos perfis de eficácia e de efetividade dos tratamentos e de resistência para emprego racional dos medicamentos antimaláricos ${ }^{6}$.

Segundo dados do M inistério de Saúde(MS), mais de $60 \%$ do território nacional possui condições ecológicas propícias para a transmissão da malária, sendo $99 \%$ dos casos na região amazônica, onde o A. darlingi éo principal vetor ${ }^{7}$. Tendo em vista que os casos de malária diagnosticados hoje no Brasil são preponderantemente por P. vivax e, em menor grau, por $P$. falciparum, o protocolo oficial aborda, principalmente, esquemas de tratamento para infecções por essas espécies?.

Qualquer esquema terapêutico deve ser seguro, eficaz, disponível, ser aceito pelas populações e possuir custo acessível para os governos ${ }^{5}$. No entanto, ainda que os medicamentos estejam disponíveis para todos, com qualidade assegurada, caberá ao sistema de saúde prover as condições para real ização do tratamento, em especial estrutura física e recursos humanos capacitados no diagnóstico, prescrição, dispensação e seguimento, de modo a oportunizar a adesão, considerada medida de sucesso terapêutico ${ }^{8}$.

Parece oportuna a discussão referente à qualificação dos recursos humanos para a atenção à malária. A importância da atuação dos profissionais de saúde nos desfechos terapêuticos observados no paciente tem sido discutida nas enfermidades crônicas e percebidas como complexas, tais como aids, em que o cuidado está centrado na figura do médico $0^{910}$. Porém, na malária, as questões se passam de forma diferente. Como, no país, as atividades de combate às endemias têm contado com a participação detécnicos eagentes de saúde ${ }^{11,12}$, investiga-se a possibilidade de que estes profissionais estejam atuando não apenas na realização de exames diagnóstico, mastambém edefacto, na prescrição, dispen sação eseguimento do tratamento antimalárico.

Algunsestudos apontam para problemasquanto a práticas e conhecimentos dos profissionais de saúde envolvidos no tratamento da malária como fatores determinantes para o cumprimento do re gime terapêutico ${ }^{13}$. Falhas na prescrição e na dispensação de medicamentos antimaláricos estariam relacionadas a aumento de resistência ${ }^{14}$.

Em 2005, o Projeto RAVREDA - Rede Amazônica de Vigilância da Resistência às Drogas Antimaláricas -, investigando a resistência a antimaláricos em unidades sentinela, apontou para a necessidade de esclarecimento de certos aspectos específicos do tratamento, que estariam influenciando os desfechos de forma preponderante. Esses aspectos estariam relacionados à aceitação dos profissionais de saúde aos esquemas terapêuticos oficiais $\varsigma^{7,15}$, o que, por sua vez, poderia ser fator de comprometimento da adesão.

No Brasil, o Programa Nacional de Controle da M alária (PNCM) adota e preconiza as condutas constantes no Manual Terapêutico da Malária (M TM ), o protocolo oficial - edição de 2001 - atualmente em revisão. 0 enfrentamento da doença é alicerçado pelo binômio diagnóstico precoce tratamento ${ }^{7,16}$. 
O objetivo deste trabalho foi elucidar práticas, conhecimentos e percepções acerca do tratamento eatitudes dos profissionais envolvidos no tratamento da malária, frente ao esquema terapêutico proposto pelo M inistério da Saúde, em seis municípios de alto risco região amazônica.

Este estudo é parte integrante do Projeto M afalda (Modelo de Avaliação para Assistência Farmacêutica em Endemias Focais na Amazônia Legal, Brasil: Prescrição, Dispensação e Adesão ao Tratamento de M alária não Complicada por P. vivax e P. falciparum em municípios de alto risco), tendo recebido aprovação do Comitê de Ética em Pesquisa da ENSP/Fiocruz ${ }^{17}$.

\section{Abordagens conceituais}

0 tratamento correto eoportuno da malária visa assegurar a cada paciente uma cura clínica rápida e duradoura, encurtar os episódios clínicos, reduzir a ocorrência de anemia associada à malária, além de impedir o desenvolvimento da re sistência aos medicamentos antimaláricos ${ }^{4}$. Desta forma, reduzirá sua duração e evitará a ocorrência de complicações e morte.

Segundo Silva et al. ${ }^{18}$, o controle da doença encontra diversos obstáculos, entre eles as questões ligadas à terapêutica. 0 tratamento é longo eemprega combinações de diversos medicamentos, em diferentes intervalos de administração, com o objetivo de enfrentar o plasmódio nas múltiplas fases de seu ciclo no organismo e de impedir surgimento de resistência do agente à terapêutica. Além disso, até hoje não existe um tratamento igualmente específico para as duas espécies de plasmódio mais prevalentes no Brasil (P. vivax eP. falciparum), sendo ainda difícil diferenciar clinicamente a infecção por uma ou outra espécie ou por ambas.

Assim, a escolha do melhor esquema terapêutico deve ser feita de forma criteriosa, por quem realmente conheça a doença, seus determinantes eas possibilidades de complicações em cada paciente. Tanto para o diagnóstico quanto para a prescrição, consideram-se o tipo de plasmódio, a classificação clínica do paciente - se malária não complicada ou malária grave - e a evolução da doença em grupos especiais a que o paciente possa pertencer, como crianças, idosos e mulheres grávidas ${ }^{19-21}$.

A procura pelo atendimento é outro problema que atinge uma grande parcela da população sob risco de malária, tanto pela escassez de re cursos nos recantos mais distantes da região amazônica quanto por força das dificuldades de deslocamento para sítios onde existam unidades ou centros de saúde. Estes fatores podem determinar intervalo importante entre a manifestação da doença e a coleta de lâmina, diagnóstico e tratamento ${ }^{22}$.

0 protocolo terapêutico para a doença, no Brasil, é representado pelo $M$ anual Terapêutico da M alária (M TM ), publicação do M inistério da Saúde, de 2001, que está, atualmente, sob revisão. O MTM pretende ser o guia para procedimentos dediagnóstico, prescrição e cuidados em malária, para agentes de endemias e profissionais de saúde, como enfermeiros, farmacêuticos e médicos. Traz questões gerais sobre a doença e sobre terapêutica, inclusive a farmacologia de diversos antimaláricos, esquemas de tratamento mais recomendados, questões sobre a resposta à terapêutica e a profilaxia7. Recentemente, foi introduzido um encarte, complementar ao M TM, que faz referência a um novo tratamento para $P$. falciparum ${ }^{23}$, aplicado em algumas regiões da Amazônia.

A orientação sobre o tratamento é parteintegrante da prescrição médica ${ }^{24}$ e também normativa do processo de dispensação de medicamento ${ }^{25}$. Além disso, a prescrição, a dispensação e a orientação adequadas constituem-se como pilares da adesão ao tratamento ${ }^{8,26}$.

As condições apresentadas pelos indivíduos sob maior risco de adoecimento por malária possuem características semelhantes. Grande parte viveno meio rural, apresenta baixo nível de escolaridade, possui, muitas vezes, condições insatisfatórias de moradia e higiene e recursos escassos para gastos com aspectos básicos de sobrevivência, como saúde e alimentação ${ }^{22}$.

Somam-se a este perfil socioeconômico, notadamente representado por estratos mais pobres da população, as dificuldades já referidas quanto a tratamento e acesso a serviços. 0 procedimento de orientação sobre a malária, incluindo prevenção, evolução da doença etratamento, deve considerar especificamente 0 contexto onde se dá a endemia, de modo a ser efetivo ${ }^{27,28}$. Percebe-se também que a oportunidade para realização da orientação ao paciente não pode ser desperdiçada, sendo absolutamente essencial para as ações de controle ${ }^{29}$.

\section{Metodologia}

Realizou-se um estudo de caso, utilizando-se metodologias quantitativa e qualitativa, esta úl- 
tima focada na análise de conteúdo. Esta análise constitui-se em um conjunto de técnicas complementares entre si, que visa analisar sistemática e objetivamente o conteúdo de comunicações ou mensagens e que permite deduzir, de forma lógica e justificada, as condições que levaram à produção das mesmas ${ }^{30}$.

Os dados foram coletados em quinze unidades de saúde de seis municípios de alto risco (Índice Parasitário Anual - IPA > 50) para malária não complicada, na Amazônia Legal, entrejaneiro e fevereiro de 2007. Esses municípios, eleitos de acordo com critérios estabelecidos no protocolo do Projeto $\mathrm{M}$ afald da ${ }^{17}$, não constituem amostra representativa da Amazônia Legal e sim Iocais com alta prevalência de malária não complicada (pelo menos 7.000 casos/ano), com cobertura por Programa de Saúde da Família (PSF) ou Programa de Agentes Comunitários de Saúde (PACS) e com adoção obrigatória do protocolo nacional do PNCM. Uma vez cumpridos estes requisitos, foram escolhidos por critério populacional: um município com mais de 1.000 .000 habitantes ( $M$ anaus, $A M$ ), um com população entre 100.000 e 1.000 .000 (Porto Velho, RO), dois com população variando de 30.000 a 100.000 (Ariquemes, RO e Cruzeiro do Sul, AC) e dois pequenos, com menos de 30.000 habitantes (Presidente Figueiredo, AM e Rodrigues Alves, AC).

As unidades visitadas foram aquelas que, em cada município, concentravam o maior número de casos atendidos de malária não complicada, de acordo com informações das Secretarias Municipais de Saúde, previamente contatadas. Aos secretários municipais de saúde e aos gestores de cada unidade foi solicitada autorização por escrito, para realização da pesquisa.

Sessenta e três (63) de sessenta e quatro profissionais, prescritores e dispensadores para malária, envolvidos na rotina de atendimento, concordaram em participar do estudo e responderam a entrevistas semi-estruturadas organizadas em roteiros específicos para cada ator. Todos os demais manifestaram sua concordância mediante termo de consentimento. As entrevistas foram gravadas e transcritas. Foi garantido o si- gilo quanto à identificação dos sujeitos. Paratanto, omitiu-se, além do nome, a referência nominal ao município de origem dos profissionais entrevistados.

Foram investigados o tempo de experiência dos profissionais e o tempo de treinamento dos mesmos para atendimento de pacientes com malária. Estes dados, quantitativos, foram descritos por meio de medidas de tendência central - média e mediana - por município, demodo a forne cer características quanto ao perfil dos profissionais. Para tanto, foi utilizado o aplicativo SPSS-10 (Statistical Packagefor the Social Sciences-10).

$\mathrm{Na}$ abordagem qualitativa, realizou-se análise de conteúdo temática por meio de categorização. Enquanto um grupo de pesquisadores realizava a busca de elementos nas falas a fim de criar categorias analíticas, um outro grupo classificava o material a partir de organização teórica prévia. A utilização desses dois processos de trabalho objetivou a identificação, codificação e validação das categorias e subcategorias, estabelecidas ao final do processo, em consenso ${ }^{30}$.

Ao final da categorização, as seguintes categorias analíticas foram identificadas: características gerais do atendimento, utilização do protocolo e orientação ao paciente.

\section{Resultados ediscussão}

0 perfil dos sessenta e três profissionais entre vistados é caracterizado por alguma amplitude entre as médias emedianas de tempo de atuação, por município (Tabela 1). Nos menores municípios, os profissionais de saúde atendem malária há menos tempo e Manaus e Porto Velho assemelham-se em relação à média e à mediana. No entanto, observa-se que o município de Ariquemes apresenta tempo diferenciadamente maior de atuação de profissionais.

Entre todos os entrevistados, 61 eram agentes de endemias, havendo apenas uma enfermeira e um médico. Tendo em vista que as visitas ocorreram em municípios pequenos e naqueles de médio e grande porte com alta incidência de

Tabela 1. Tempo de trabalho (anos) de profissionais que atendem malária. Municípios da Amazônia Legal, 2007.

\begin{tabular}{lccccc}
\hline M unicípio & Ariquemes & Rodrigues Alves & Cruzeiro do Sul & M anaus & Presidente Figueiredo \\
\hline média & 17,7 & 1,7 & 4,5 & 8,1 & 7,3 \\
mediana & 20 & 1 & 2 & 4 & 3 \\
\hline
\end{tabular}


malária, esperar-se-ia maior participação total demédicos, enfermeiros efarmacêuticosna atenção à doença.

0 manual de treinamento dos agentes no controle da malária prevê o envolvimento tanto deagentes comunitários de saúde como de agentes de endemias ${ }^{12}$ no combate à doença. $\mathrm{O} \mathrm{MS}$ recomenda ainda a integração das ações de ambos em áreas endêmicas ${ }^{31}$. No entanto, observou-se que, dos agentes entrevistados, 100\% eram agentes de endemias. Eles são percebidos, nos municípios, pelos demais agentes, comunitários e do PSF, como sendo específicos para o combate à malária, além de possuírem vínculo diferenciado dos demais.

No caso de tempo de capacitação (Tabela 2), observa-se que a diferença entre mediana e mé dia, em Manaus, Porto Velho eAriquemes, éconsiderável. Este dado pode indicar diferença de horas de treinamento entre os profissionais que atendem nestes municípios, apontando para falta dehomogenei dade na capacitação. Não houve informação quanto ao tempo de treinamento formal no município de Presidente Figueiredo.

Embora não haja tempo regulamentado para treinamento, estima-sequeuma capacitação permanente seja a mais adequada ${ }^{32}$. No entanto, aventa-se que a qualidade e não apenas o tempo em horas de treinamento seja determinante para 0 bom atendimento aos pacientes. Nota-se que o município de Cruzeiro do Sul é o que apresentou maior tempo de treinamento dos profissionais. 0 acréscimo de horas de treinamento no município pode estar relacionado a estudo clínico envolvendo artesunato, quese desenrolava no município por ocasião da visita.

\section{Características gerais do atendimento}

0 atendimento à malária é fortemente pautado pela notificação. Ela é entendida, corretamente, aliás, pelos agentes e profissionais envolvidos, como ação essencial e necessária, a primeira de uma sériede procedimentos que devem se encadear: "Primeiro a gente faz a notificação.
Quevem do nosso instituto, queédo M inistério da Saúde. Faz toda investigação, desde a casa onde mora o paciente, idade, o endereço, o local provável de infecção. A gente pergunta quantos dias ele esteveno local. Caso elesaiu da cidadepro interior. E depois disso faz a coleta. M anda aguardar uma hora, esse paciente, e espera pelo resultado, positivo ou negativo" . (Agente)

Os agentes estão absolutamente al inhados ao sistema de notificação, pelo SIVEP (Sistema de Vigilância Epidemiológica). A notificação éfeita havendo ou não confirmação do diagnóstico e do tipo de malária. Estima-se que as anotações complementares sejam efetuadas, atéporque são indispensáveis para a dispensação do tratamento adequado ou para o encaminhamento do paciente em caso negativo.

A seguir, na hierarquia de elementos do discurso, vem o diagnóstico, ainda que ele, teoricamente, devessepreceder a notificação. 0 correque, como relata o médico abaixo, em áreas endêmicas, muitas vezes o próprio paciente já sabe que tem a doença eo médico conseguediagnosticá-la clinicamente: " 0 diagnóstico, por se tratar deuma área endêmica, é feito (por) busca ativa e tudo mais, muitos casos já chegam com o diagnóstico feito na lâmina do microscopista na área rural. Já, todos os pacientesjá sabendo, conhecendo a malária chegam falando, 'doutor, eu estou com malária'". (M édico)

De qualquer forma, o binômio diagnósticonotificação é a base da atenção à malária, pre sente nos discursos de todos os entrevistados. É interessante notar que a preocupação com notificação, diagnóstico etratamento está onipresente no sistema de saúde, como destacam Rocha et al. ${ }^{26}$. 0 Manual Terapêutico da Malária reforça este paradigma ${ }^{7}$. A promoção e a prevenção estariam subentendidas como de competência do doente ${ }^{26}$.

$\mathrm{Na}$ vigência deste paradigma, fal ha, falta ou demora no diagnóstico e, por conseguinte, no tratamento, resultariam na exclusão do doente à atenção especializada para malária e em consequências mórbidas ${ }^{33}$. Outra questão importante

Tabela 2. Tempo de capacitação (horas) de profissionais para atender malária. Municípios da Amazônia Legal, 2007.

\begin{tabular}{lcccccr}
\hline Município & Ariquemes & Rodrigues Alves & Cruzeiro do Sul & Manaus & Presidente Figueiredo & Porto Velho \\
\hline média & 266,7 & 200,0 & 413,5 & 174 & $\mathrm{SI}$ & 133,3 \\
mediana & 160 & 200 & 480 & 40 & $\mathrm{SI}$ & 300 \\
\hline
\end{tabular}

Sl - sem informação. 
neste cenário diz respeito a dificuldades no diagnóstico. Abdon et al. ${ }^{34}$ destacam, por exemplo, que a malária por $P$. vivax pode sofrer de imprecisão diagnóstica, levando a conclusões que irão causar terapêutica imprópria.

A atenção à malária nos municípios visitados éfeita, quase que exclusivamente, por agentes de endemias. São treinados, pelo MS, para a notificação, o diagnóstico laboratorial (exame de gota espessa), dispensação de antimaláricos, orientação everificação de cura ${ }^{12}$ : “0 paciente que chega aqui (na unidade), écol etada a lâmina do paciente, se der positivo, dependendo da área aonde ele reside, tem o agente, que aqui é tudo dividido por território, então 0 agente já conhece a pessoa, a localidade, vai lá deixar medicamento, tudo completo. E tenta o máximo possível acompanhá-lo atéo final, ou seja, deixa a primeira dose. Depende também muito do paciente. Porque, às vezes, 0 pacienteéresponsável ea gente sabe que eletoma 0 medicamento atéo final. N essescasos, o agentedeixa o medicamento todo" . (Agente)

Tendo em vista os elementos já destacados, a situação, ainda que representativa da estratégia possível para o controle de endemias no nosso território, traz consequências previsíveis. É deixada ao agente de endemias, com variáveis tempos de atividade e de capacitação, a decisão clínica sobre o paciente e sobre a capacidade do mesmo em levar ao fim seu tratamento.

U ma importante consequência direta da tomada de decisão pelos agentes está na falha de tratamento antimalárico em situações em que os únicos diagnósticos possíveis são o clínico e o epidemiológico ${ }^{7,12}$. Nestas situações, é preconizado que o tratamento seja feito considerando a malária mais prevalente na área de origem do paciente. A omissão destetipo de ocorrência nas falas pode ser indicativa, não da ausência dessas situações, dada a prevalência da malária nas áreas visitadas, mas da incapaci dade dos agentes em lidar com a situação.

Em um dosmunicípios, foi citada, pelosagentes, que a presença do médico no atendimento à malária seria necessária apenas nos casos mais graves, após triagem de casos. Porém, o médico não está presente na aten ção diária aos pacientes com malária, em quaisquer dos seis municípios de alta incidência visitados. Como se discute acima, a ausência do médico traria prejuízos consideráveis à qualidade da atenção.

A pesar de praticamente ausente da atenção à malária nos municípios visitados, a presença ea participação do médico é percebida como de importância pelos agentes: "Se ele não tá bem, a gente encaminha ao médico. Pra ser melhor orientado" . (Agente)

Em vários estudos, tem ganhado destaque a relevância da adesão do paciente ao tratamento. Entretanto, esta adesão tem como pressuposto, entre outros, a prescrição médica ${ }^{8,26,35}$. Como, no Brasil, a lei confere apenas a médicos ea odontólogos esta permissão ${ }^{36}$, no caso da assistência à malária, em não atuando o médico, a prescrição estará também ausente.

Da mesma forma que a adesão está condicionada ao determinante prescrição e orientação, está também vinculada à dispensação ${ }^{14}$. A dispensação consiste em atividades de separação, conferência, entrega e orientação sobre os medicamentos e sobre 0 tratamento ${ }^{37}$. $\mathrm{Na}$ atenção à malária, existeclara referência à importância que o diagnóstico laboratorial teria para a dispensação dos medicamentos: "A gentefaz a notificação, faz a coleta, que é furar o dedo, pra gente pegar as gotas (de sangue), depois passa no processo de coloração, depois vai pro laboratório. Pro microscopista, pra fazer análise. E se der a malária, a gente faz a dispensação da medicação e as orientações". (Agente)

Outro importante pressuposto para a adesão éa dispensação. Embora presentena fala dos agentes entrevistados, apenas um município qualificou a dispensação, caracterizando-a como sendo a conferência da quantidade dos medicamentos. A fala citada apresenta uma concepção recorrente entre os entrevistados, que entendem a dispensação como a simples entrega dos medicamentos.

Que implicações teria a falta de dispensação completa sobre o tratamento? Uma vez que na atenção à malária não existe prescrição, a priori a dispensação estaria prejudicada. Ainda, não se menciona a presença de farmacêuticos nos serviços. Dada à complexidade do tratamento de malária, este profissional desempenharia importante papel na orientação e no seguimento dos pacientes. É digno de nota que as características relacionadas aos medicamentos, como doses, esquemas e sua duração, efeitos adversos e interações, são consideradas importantes causas para sucesso ou insucesso do tratamento antimalárico 14,18,22,34.

\section{Utilização do protocolo}

O Manual Terapêutico da M alária (M TM) é o principal instrumento normativo de condutas e terapêuticas para malária, estabelecido pelo PNCM ${ }^{38}$. Segundo a fala dos entrevistados, é também, sem qualquer dúvida, o norteador das práticas dos agentes de endemias nos municípi- 
os visitados. Neste sentido, o papel oficial do MTM , como fonte única para subsidiar a decisão clínica, écumprido: “A forma queé feito todo o tratamento é baseado em cima do manual terapêutico. É feito tudo por lá. Tudo tem que se fazer por ele. Porque ninguém tem informação profissional para mudar o tipo de tratamento que é feito pelo manual". (Agente)

"Nós só damosa medicação mediantea tabela, que é padronizada, pelo Ministério da Saúde". (Agente)

No entanto, algumas questões influem na efetividade desta utilização, ou seja, embora o M TM contenha as informações consideradas adequadas para o tratamento, a forma de veiculação e o preparo dos agentes redundam em comprometimento dos resultados de aplicação .

O MTM contém diversos esquemas tabelados, tanto em relação ao tipo de paciente quanto em relação ao tipo de malária. Esses esquemas contêm fármacos utilizados no país, mas em doses que não se reproduzem em formas farmacêuticas produzidas e disponíveis nos serviços. No momento da dispensação, por exemplo, quando o MTM traz " $11 / 2$ " comprimido ou "1/4" de comprimido, a única conduta adequada seria 0 fracionamento da forma farmacêutica na unidade, por profissional habilitado para tal39. Pode ser que, quando seja feita a entrega dos medicamentos, o paciente receba o total de comprimidos sem que seja considerado o fracionamento, o que certamente importará em risco.

Por outro lado, o M TM contém informações terapêuticas eesquemas deuso para medicamentos que não são utilizados no Brasil| ${ }^{6}$. Esta informação, sem dúvida útil em situações específicas, como por exemplo doações, pode confundir o agente no momento do atendimento.

Por fim, o M TM , na edição de 2001 (vigente), não traz todos os medicamentos presentemente utilizados no país para o tratamento de malária. Em algumas regiões da Amazônia (mas não em todos os estados ou municípios), o PNCM está introduzindo novas opções terapêuticas. É o caso da combinação artemeter +lumefantrina, comercializada com o nome de marca Coartem ${ }^{\circledR}$.

De modo a dirimir dúvidas quanto à utilização deste medicamento, foi lançado um encarte, em 2006'23, pelo PNCM, com os esquemas novos, que complementaria as informações do MTM 2001. Para os agentes entrevistados, este documento é compreendido como um "substituto" oficial ediferentedo manual: "Coartem tem no manual sim. Tem um outro manual. É outro manual". (Agente)
“H oje, Coartem. Anteriormente, antes do Coartem tinha mefloquina. Anteriormente era como 0 esquema do M inistério. Hoje nós fazemos o Coartem. Só o Coartem. Aí muda apenas a dose, dependendo da faixa etária, peso". (Agente)

No entanto, no cotidiano dos atendimentos, pode-se perceber a aplicação indistinta do M TM 2001 edo encartepublicado em 2006. Como trazem esquemas diversos para as mesmas indicações (malária por P. vivax, por P. falciparum e mista, na gestação), especulam-se as possibilidades de erros de aplicação dos esquemas.

É interessante ressaltar também que, em relação ao Coartem ${ }^{\circledR}$, a referência se dá, sempre, com o nome do produto e não com o nome dos princípios ativos, como é o caso de outros medicamentos presentes no manual. 0 fornecimento futuro de produtos com diferente nome de marca ou genérico poderá suscitar problemas.

Considerando todas as questões que envolvem os esquemas terapêuticos, observa-se o erro frequente de conduta dos agentes na prática diária, principalmente para tratamento de $P$. falciparum, que ocasiona a forma mais grave de malária: "Para falciparum, nós dispensamos o quinina mais doxiclina ea primaquina no oitavo dia. $\mathrm{E}$ para malárias falciparum resistente, nós dispensamos a mefloquina, chamada-se de dose única, mais primaquina por sete dias". (Agente)

"Estamos usando o Coartem. Para falciparum. M as fora disso, se o paciente tomar, usa a primaquina. 0 último dia (que é o) terceiro, dia é três primaquina. Todo paciente". (Agente)

"Ah, nós só temos os doisti pos de medicamento pra falciparum atualmente. É o quinino e a dose única, queéa mefloquina. M efloquina nósusamos quando a malária é resistente". (Agente)

0 esquema recomendado originalmente no M TM 2001 para P. falciparum é: quinina em três dias + doxiciclina em cinco dias + primaquina no sexto dia. 0 encarte preconiza o uso do Coartem $\AA$ como primeira escol ha para tratamento de P. falciparum em três dias. 0 tratamento alternativo érepresentado por mefloquina em doseúnica, seguida por primaquina no segundo dia ${ }^{7,23}$. Outra alternativa é quinina em sete dias.

Em relação à malária por $P$. vivax, os medicamentos parecem ser mais conhecidos pelos agentes; no entanto, ainda pode-se observar faIhas importantes na percepção quanto à combinação dos mesmos e aos possíveis esquemas empregados: "Para vivax, nós dispensamos o me dicamento à base de cloroquina e primaquina" .

"Só a cloroquina e a primaquina. É a única alternativa quetem. É o único tratamento". (Agente) 
O esquema recomendado pelo M TM 2001 e também no encarte de 2006 para P. vivax é de cloroquina em três dias + primaquina em sete dias. O M TM 2001 preconiza esquema alternativo para P. vivax, em crianças apresentando vômitos ( cápsulas retais de artesunato em quatro dias e primaquina em sete dias). No entanto, nem crianças até seis meses de idade nem gestantes podem usar primaquina.

Estima-se que a variação de tratamentos nos diversos municípios, ainda que se admitindo as orientações peculiares em cada um, reflita situação não desejável do ponto de vista do tratamento e do programa nacional, podendo contribuir como determinante de resistência.

Um dos motivos possíveis de fal has de informação na prática diária dos agentes pode ser a errática presença do M TM 2001 e/ou do encarte 2006 nas unidades visitadas: "Temos. Em todas as unidades. Lá nas comunidades do município, temos em todas elas. E também na equipeenviada no campo a gente tem também". (Agente)

"Não. Tenho e não tenho, porque quando tem alguma dúvida, a gente vai lá com a doutora bioquímica e ela informa pra gente. Ela mesmo vêno manual alguma coisa. Não tenho (o MTM)". (Agente)

\section{Orientação ao paciente}

As orientações ao paciente, nasfalas dosagentes entrevistados, mostra preocupação com alguns pontos específicos, ainda que resultem em informações tanto certas quanto erradas. U ma das falas mais recorrentes é quanto à importância de obediência a horários eregimes terapêuticos: "Por exemplo, uma informação que é muito importante. É a questão do horário". (Agente)

"M as a principal informação que nós passamos para ele é a questão da sequência correta". (Agente)

É interessante ressaltar que, enquanto absolutamenteprocedente, esta preocupação tanto em informar horários quanto que medicamentos tomar e em que dias, se contrapõe aos erros observados no relato sobre os esquemas terapêuticos. De sorte que se pode chegar a uma situação inusitada - reiterar horários e sequências para medicamentos equivocados. Silva et al. ${ }^{22}$ denotam que a falta de explicações corretas seria uma das "falhas técnicas" na condução de casos de malária.

Uma outra vertente de orientação abordada pelos agentes diz respeito à alimentação, à ingestão de bebidas alcoólicas, à necessidade repouso e de cuidados com a exposição ao sol: “O rientamos. Orientamos, pra ter um repouso depois que tomar a medicação. Não usar bebida alcoólica. De primeiro era aconselhado tomar acompanhado com leite, mas hoje a gente teve um treinamento que não pode, porque o leite, ele corta o efeito da medicação também. M as damos orientação pra ele tomar aquela medicação depois que ele se alimentar. Tiver alimentado, então melhor perto da hora de ele dormir também. Queéuma hora que elejá vai repousar. Elenão tá pegando sol, não tá trabalhando no trabalho pesado, até ele, pelo menos, éa me Ihor orientação que a gente dá, não é"? (Agente)

Ainda que a preocupação geral dos agentes seja legítima, ela não está fundamentada no M TM , onde as orientações são medicamento-específicas. Por exemplo, no caso da mefloquina, a orientação oficial é para não tomar antes de dormir. Embora seja aconselhável tomar medicamentos com estômago cheio, na maior parte dos casos, há exceções importantes. Os alimentos podem impedir a absorção de alguns medicamentos ou interagir com princípios ativos, causando efeitos danosos e permanentes. Esteé o caso da ingestão deleitecom tetraciclina eseus derivados, queleva à quelação do cálcio com hipoplasia óssea e permanenteescurecimento dos dentes, razão pela qual não é recomendada para gestantes (feto em desenvolvimento), lactentes e crianças ${ }^{40}$.

Os agentes, ao fazerem recomendações de cunho muito generalizado, podem estar seguindo as determinações do $M$ anual dos Agentes de Endemias ${ }^{12}$, que orienta superficialmente, recomendando apenas não tomar medicamentos em jejum e evitar bebidas alcoólicas.

0 discurso dos agentes seressentedereferências consistentes aos efeitos adversos dos antimaláricos. São efeitos importantes, que podem evoluir para quadro de desidratação e têm consequências determinantes na adesão ao tratamento $0^{8,22,35}$.

$N$ as falas dos agentes, observou-se três argumentos recorrentes em muitas entrevistas: não tomar antimalárico quando houver febre, em caso de febre utilizar antitérmico e utilizar antitérmico quando não dispuser de antimalárico: "Para estar alimentado, sem febre. Quando tiver febre não tomar". (Agente)

"Q ue a gente sempre coloca pro paciente, caso apresente a febre, pra ele tomar antitérmico" . (Agente)

“Então a gente sempreorienta queelenão tome dipirona, sequencialmente, mas apenas quando ele não tiver o remédio". (Agente)

$\mathrm{N}$ ão se recomenda, nas normas orientadoras, que se trate febre inespecífica como malária. 
$\mathrm{O}$ diagnóstico é norteador do tratamento. $\mathrm{Na}$ falta de diagnóstico laboratorial, deve ser feito diagnóstico clínico e epidemiológico, como já se observou. No entanto, esta informação pode estar sendo traduzida, pelos agentes, como o inverso - não se tratar malária sem diagnóstico laboratorial, em caso de febre.

A indicação para utilização de antitérmicos, na presença de febre, existe apenas para casos de vômitos associados à utilização de mefloquina, ocasião na qual também podem ser indicados antieméticos ${ }^{7}$. Na recomendação para uso concomitante de antimalárico e antitérmico, poderia estar ocorrendo generalização e má interpretação destas orientações, uma vez que outras condutas com relação à febre não estão documentadas nos manuais orientadores do $\mathrm{M} \mathrm{S}^{7,12}$, tampouco no Formulário Terapêutico Nacional ${ }^{40}$. No entanto, sempre que for necessária, a conduta devese dar na vigência do tratamento enão como substitutivo. Estudo realizado em Uganda ressalta que, entrepacientesecuidadores, existepercepção de que febre e malária se confundem ${ }^{41}$.

A substituição do uso de antimaláricos por antitérmicos na vigência de febre, tampouco a indicação dealgum antitérmico específico, como dipirona, por exemplo - fármaco que possui perfil de segurança duvidoso ${ }^{42}$ - estão citados nas fontes oficiais de conduta em caso de malária.

H ouve interessante coincidência entre as falas dos agentes nos municípios visitados e os re latos presentes em estudos sobre percepções, conhecimentos e práticas de pacientes e trabal hadores em saúde atendendo malária nos continentes africano e sul-americano: "Aí, depois da medicação. Tomar bastante líquidos, suco. M uito doce também. Que isso ajuda muito o fígado. Restaurar. Porque devido à medicação que é bastante forte". (Agente)

"Durante o tratamento, ele não deve (cometer) excesso. Frutas ácidas, (comidas) muito gordurosas e (bebidas) alcoólicas". (Agente)

Os medicamentos antimaláricos são percebidos tanto como agentes de cura como promotores de dano ${ }^{13,41,43}$. A percepção da necessidade de proteção ao fígado se expressa por meio da prescrição de condutas "protetoras", pela ingestão de alimentos açucarados, líquidos e sucos ${ }^{41}$. Em comunidades rurais na Colômbia, a proteção se expressa por uma dieta livre de gorduras, para prevenir recaídas ${ }^{44}$.

Os agentes consideram a necessidade de informar o paciente da importância do retorno para coleta da lâmina de verificação de cura (LVC). A recomendação é adequada e segue os manuais.
Além disso, estaorientação ajuda a esclarecer se, ao final esperado do tratamento, com cessação dos sintomas, houve cura do paciente: "E depois que terminar a medicação, colher nova lâmina pra ver se realmente ele tá, tá curado. A LCV, que a gente chama de lâmina de verificação de cura. Essa éa orientação quea gente dá pro paciente". (Agente)

Segundo a literatura, o abandono do tratamento antimalárico écomum ${ }^{18,34}$. Deacordo com o relato dos agentes, o abandono do tratamento é frequente nas áreas visitadas: "Porque o quarto dia, o quinto dia, aí a pessoa não está mais sentindo dor de cabeça, nada. Aí não quer mais tomar". (Agente)

$\mathrm{O}$ abandono acontece, normalmente, com a melhora dos sintomas ${ }^{18}$. Outra explicação pode ser a falta de informação e a alta incidência dos efeitos adversos provocados pelos medicamentos- especialmente, nestecaso, pelos antimalári$\cos ^{8,26}$. Duarte e Gyorkos ${ }^{16}$ sugerem que a duração e a complexidade destes tratamentos seriam fortes causas para 0 abandono.

O nível socioeconômico e cultural dos pacientes tem consequências sobre sua compreensão sobre o tratamento, comprometendo a adesão; os determinantes socioeconômicos são apontados, por vários autores, como barreiras importantes para a adesão 05,46 .

A efetividade do tratamento da malária, no Brasil, está subordinada, assim, não apenas à situação epidemiológica, a ações de combate ao mosquito, à disponibilidade dos medicamentos edosserviços de saúdee ao treinamento dosagentes, mas também ao paciente que recebe e assimila a informação: "Mas devido a maioria das pessoas que contraem a malária, no garimpo, seja na área rural, seja nas fazendas, são pessoas de baixo nível de informação. São pessoas humildes que vêm até nós, pegam o medicamento, você explica, mas ele virou a esquina, ele já esqueceu. Então ele vai tomar pelo 'disse'. 0 vizinho lá 'disse' que é assim que toma. E aí quase dez, quinze dias, ele retorna pra fazer a lâmina de verificação de cura e tá com malária. Aí vocêpergunta pra ele, 'o senhor tomou o remédio direitinho como eu te falei?' 'Ah, tomei tudo'. Mas não é pra tomar todo o medicamento! E sim, tomar os medicamentos na hora certa. Ele já tava, não com uma malária nova, uma malária mal tratada, mal curada. Isto acontece inúmeras, inúmeros casos, elesretornam sabe? Três, quatro vezes, o mesmo paciente, a droga já não tá fazendo mais efeito. Aí o quêque eu faço? Eu trago esse paciente pra vim fazer o tratamento? $\mathrm{N}$ ão vou te dar o medicamento pra vocêlevar pra casa. Você vai vim tomar comigo aqui no balcão. E eles vêm, 
e tomam esurte efeito. Agora eu não sei se o medicamento não faz efeito lá com eles, ou se elesjogam o medicamento fora" . (Agente)

Percebe-se, pela situação apresentada nos municípios visitados, de alto risco, que o treinamento dos agentes deixa fal has importantes. Porém, inegavelmente, a lida diária dos agentes no cuidado permite que eles construam, em alguns momentos, uma crítica sobre seu processo de trabalho, propondo alternativas. Elas surgem menos a partir do conhecimento estabelecido e formalmente apresentado aos agentes e mais do senso comum, das experiências cotidianas.

\section{Consideraçõesfinais}

A utilização do discurso dos agentes de endemias nos municípios visitados permitiu apreender os significados que permeiam suas práticas, conhecimentos e percepções sobre malária e sobre 0 tratamento.

O conteúdo das entrevistas foi analisado por meio de categorização, mediantea técnica deBar$\operatorname{din}^{30}$. Outrossim, a categorização feita por duas vertentes complementares promoveu o refinamento da análise quanto aos temas pertinentes ao estudo. Foi interessante observar que se consolidou, no exercício da categorização, a explanação teórica da autora quanto às possibilidades de aplicação do método Bardin ${ }^{30}$, o que representou um ganho conceitual à análise.
Os profissionais que atendem malária nos municípios de alto risco na Amazônia Legal estudados são, em quase total maioria, agentes de saúde, denível médio ou auxiliar, treinados especificamentepara estefim. A ausência do médico foi percebida como uma constante nas unidades de saúde visitadas. 0 treinamento parece ser focado no uso dos manuais do MS, na notificação, no diagnóstico e na entrega do medicamento mediante confirmação de caso. Há uma ênfase na rotina, com pouca reflexão sobre as especificidades do tratamento e da doença. Percebe-se ainda um isolamento das práticas estabelecidas para malária das demais iniciativas de cuidado, como por exemplo, da atenção básica, nesses municípios.

Por outro lado, esses agentes de endemias percebem-se limitados em relação a sua capacidade de enfrentamento em situações especiais, 0 que é positivo, face à sua pouca escolaridade e a seu treinamento heterogêneo. No entanto, esses agentes foram capazes de reconhecer na população atendida barreiras importantes para a efetividade do tratamento.

Observa-se o esforço do PNCM na adequação dos profissionais envolvidos, mas estima-se que medidas mais intensivas devam ser direcionadas no sentido dequalificar eincorporar profissionais, uma vez que a variedade de condutas e práticas pode comprometer as metas do PN, levando à falta de adesão, aumento de resistência, maior morbidade em populações específicas, como gestantes e crianças, e aumento da transmissão.

\section{Colaboradores}

CGS O sorio-de-Castro e ES M iranda trabalharam na concepção teórica, execução, redação e revisão final do texto. A Esher participou da concepção teórica, redação e revisão final do texto. M R Campos trabalhou na concepção teórica, execução e redação do texto. JC Brasil, ACS Ferreira eICM Emmerick participaram da execução e redação do texto. 


\section{Referências}

1. Snow RW, Guerra CA, Noor AM, Myint HY, Hay S. The global distribution of clinical episodes of Plasmodium falciparum malaria. Nature 2005; 434:214217.

2. Panamerican $\mathrm{H}$ ealth Organization. Annual Malaria Cases and Deaths in the Americas, 1998-2006. M alaria Cases and Deaths in the Americas, 1998-2006. [site da Internet] [acessado 2008 jan 12]. Disponível em: http://www.paho.org/English/AD/DPC/CD/ mal-cases-deaths-1998-2006.pdf

3. Hay SI, Guerra CA, Tatem AJ, Noor AM, Snow RW. The global distribution and population at risk of malaria: past, present and future. Lancet Infect Dis 2004; 4:327-336.

4. World Health Organization. The use of antimalarial drugs - report of a WHO informal consultation. Geneva: WH O; 2001

5. World Health Organization. Antimalarial drug policies: data requirements, treatment of uncomplicated malaria and management of malaria in pregnancy. Report of an Informal Consultation WHO/MAL/ 94.1070. Geneva: WH O; 1994.

6. Freitas LF, Chaves GC, Wannmacher L, Osorio-deCastro CGS. Malária não-complicada por P. vivax e P. falciparum no Brasil: evidências sobre fármacos isolados e associações medicamentosas empregados em esquemas terapêuticos recomendados pelo protocolo terapêutico oficial. Cad Saude Publica 2007; 23(10):2285-2294.

7. Brasil. Ministério da Saúde. Manual de Terapêutica da M alária. Brasília: M inistério da Saúde; 2001.

8. Osterberg $\mathrm{L}$, Blaschke $\mathrm{T}$. Adherence to medication. N Engl J Med 2005; 353:487-497.

9. Malta M, Petersen ML, Clair S, Freitas F, Bastos FI. Adherence to antiretroviral therapy: a qualitative study with physicians from Rio de Janeiro, Brazil. Cad Saude Publica 2005; 21(5):1424-1432.

10. Roberts KJ, Volberding P. Adherence communication: a qualitative analysis of physician-patient dialogue. AIDS 1999; 13:1771-1778.

11. Cardoso RF, Goldenberg P. Malária no Estado do Amapá, Brasil, de 1970 a 2003: trajetória e controle. Cad Saude Publica 2007; 23(6):1339-1348.

12. Brasil. Ministério da Saúde. Ações de Controle de Endemias, M alária. Manual para Agentes Comunitários de Saúde e Agentes de Controle de Endemias. Brasília: M inistério da Saúde; 2002.

13. Mubyazi G, Kamugisha M, Kitua A, Ijumba J. Intermittent preventive treatment of malaria during pregnancy: a qualitative study of knowledge, attitudes and practices of district health managers, antenatal care staff and pregnant women in Korogwe District, North-Eastern Tanzania 2005. M alar J 2005; 4:31.

14. Depoortere E, Guthmann J, Sipilanyambe N, N kandu E, Fermon F, Balkan S, Legros D. Adherence to the combination of sulphadoxine-pyrimethamine and artesunate in the M aheba refugee settlement, Zambia. Trop M ed Int Health 2004; 9:62-67.

15. Brasil. M inistério da Saúde. Vigilância em Saúde. Situação Epidemiológica da M alária no Brasil. [site da Internet] 2005 [acessado 2006 fev 24]. Disponível em: http://www.saude.gov.br/svs
16. Duarte EC, Gyorkos TW. Self-reported compliance with last malaria treatment and occurrence of malaria during follow-up in a Brazilian Amazon population. Trop Med Int Health 2003; 8(6):518-524.

17. Osorio-de-Castro CGS, Ruiz AM, Chaves GC, M iranda ES, Mutis MS, Freitas LF, Campos M, Oliveira WP, Pimenta de Souza P. Projeto Modelo de Avaliação para Assistência Farmacêutica em Endemias Focais na Amazônia Legal, Brasil: Prescrição, Dispensação e Adesão ao Tratamento de Malária não Complicada por P. vivax e P. falciparum em municípios de alto risco. Rio de Janeiro: NAF/ENSP/FIOCRUZ; 2005.

18. Silva RSU, Pinto AYN, Calvosa VSP, Souza, JM . Esquemas terapêuticos encurtados para o tratamento de malária por Plasmodium vivax. Rev Soc Bras M ed Trop 2003; 36(2):235-239.

19. Grobusch MP, Kremsner PG. Uncomplicated maIaria. Curr Top Microbiol Immunol 2005; 295:83-104.

20. Gavazzi G, Herrmann F, Krause KH. Aging and infectious diseases in the developing world. Clin Infect Dis 2004; 39:83-91.

21. Nosten F, M cGready R, Mutabingwa T. Case management of malaria in pregnancy. Lancet Infect $D$ is 2007; 7:118-125.

22. Silva $A R$, Tauil PL, Bastos Júnior JL, Matos WB, Costa EAP, Gonçalves EGR. Aspectos da transmissão focal de malária na Ilha de São Luís, M aranhão. Rev Soc Bras M ed Trop 2006; 39(3):250-254.

23. Brasil. M inistério da Saúde. Secretaria de Vigilância em Saúde. Programa Nacional de Controle da M alária. Esquemas recomendados para o tratamento da malária não complicada no Brasil. Brasília: Ministério da Saúde; 2006.

24. Organização Mundial da Saúde. Guia para a boa prescrição médica. Porto Alegre: Artmed; 1998.

25. Brasil. Resolução n 357 de 20 de abril de 2001. Aprova o regulamento técnico das Boas Práticas de Farmácia. Diário Oficial da União 2001; 20 abr.

26. Rocha MNA, Ferreira EAP, Souza JM. Uma proposta de prevenção e controle da malária em pequenas comunidades. Rev Para M ed 2005; 19(4):47-51.

27. Suárez-M utis $M C, M$ artinez-Espinosa FE, Albuquerque BC, Coura JR. Dinâmica das doenças infecciosas e parasitárias. Rio de Janeiro: GuanabaraKoogan; 2005. p. 833-858.

28. World Health Organization. The World Malaria Re port. [site da Internet] [acessado 2006 fev 24]. Disponível em: http://rbm.who.int/wmr2005/

29. Pepe V, Osorio-de-Castro CGS. A interação entre prescritores, dispensadores e pacientes: Informação compartilhada como possível benefício terapêutico. Cad Saude Publica 2000; 16(3):815-822.

30. Bardin L. Análise de conteúdo. $3^{\mathrm{a}}$ ed. Lisboa: Edições 70; 1977.

31. Brasil. Portaria no 44/GM de 03 de janeiro de 2002. Definir as atribuições do Agente Comunitário de Saúde - ACS - na prevenção e no controle da malária e da dengue. Diário Oficial da União 2002; 03 jan.

32. Hill B, Lenzi M. Notes on the training of workers in the health sector. Cad Saude Publica 1997; 13(2):317319. 
33. Wanderley DM V, Silva RA, Andrade JCR. Aspectos epidemiológicos da malária no Estado de São PauIo, Brasil, 1983 a 1992. Rev. Saude Publica 1994; 28(3):192-197.

34. Abdon NP, Pinto AYN, Silva RSU, Souza JM. Avaliação da resposta aos esquemas de tratamento reduzidos para malária vivax. Rev Soc Bras. M ed Trop 2001; 34(4):343-348.

35. Leite SN, Vasconcellos M PC. Adesão à terapêutica medicamentosa: elementos para discussão de conceitos e pressupostos adotados na literatura. Cien Saude Colet 2003; 8(3): 775-782.

36. Brasil. Portaria $n^{\circ} 3.916$ de 30 de outubro de 1998. Política Nacional de Medicamentos. Diário Oficial da União 1998; 30 out.

37. Marin N, Luiza VL, Osorio-de-Castro CGS, Machado-dos-Santos $\mathrm{S}$, organizadores. Assistência farmacêutica para gerentes municipais. Rio de Janeiro: OPAS/OM S; 2003.

38. Brasil. M inistério da Saúde. Programa Nacional de Prevenção e Controle da M alária - PNCM. Brasília: Secretaria de Vigilância em Saúde; 2003.

39. Brasil. Ministério da Saúde. RDC n 135 de 18 de $M$ aio de 2005. Estabelece os critérios que devem ser obedecidos para 0 fracionamento de medicamentos. Diário Oficial da União 2005; 18 mai.

40. Brasil. Ministério da Saúde. Formulário Terapêutico Nacional. Brasília: Secretaria de Ciência, Tecnologia e Insumos Estratégicos; 2008.

41. M bonye Ak NS, Magnussen P. Perceptions on use of sulfadoxine-pyrimethamine in pregnancy and the policy implications for malaria control in U ganda. Health Policy 2006; 77(3):279-289.

42. Wannmacher L. Paracetamol versus dipirona: como mensurar o risco? In: Organização Pan-Americana da Saúde. U so Racional de M edicamentos: Temas Selecionados. Brasília: OPAS; 2005.

43. Schultz LJ, Steketee RW, Chitsulo L, Macheso A, Nyasulu $Y$, Ettling M. Malaria and childbearing women in Malawi: knowledge, attitudes and practices. Trop Med Parasitol 1994; 45(1):65-69.

44. Pineda F, Agudelo CA. Percepciones, actitudes y prácticas en malaria en el amazonas colombiano. Rev. salúd pública 2005; 7(3):339-348.

45. Figueiredo RM, Sinkoc VM, Tomazim CC, Gallani $M C B J$, Colombrini MRC. Adesão de pacientes com aids ao tratamento com antirretrovirais: dificuldades relatadas e proposição de medidas atenuantes em um hospital escola. Rev. Latino-am. Enfermagem 2001; 9(4):50-55.

46. Piras SS. Estudo de adesão de pacientes ambulatoriais do Instituto M unicipal Philippe Pinel a tratamentos com antipsicóticos atípicos [monografia]. Niterói: UFF; 2004.

Artigo apresentado em 10/06/2008

A provado em 08/01/2009

Versão final apresentada em 15/01/2009 